\title{
Perfil social y perfil antropométrico en el atletismo peruano juvenil de élite
}

\section{Social profile and anthropometric profile in the elite Peruvian junior athletics}

\author{
Salim Bravo \\ EAPEF, Universidad Nacional Mayor de San Marcos, Av. Germán Amezaga s/n Lima 1, Lima, Perú
}

\section{RESUMEN}

En este estudio se determinó el perfil antropométrico $(n=82)$ : forma, composición y proporcionalidad corporal, y el perfil social $(n=71)$, estatus socioeconómico, de los atletas juveniles peruanos según su nivel de rendimiento (nivel nacional y nivel internacional). Asimismo, se establecieron correspondencias entre sus especialidades, el nivel de rendimiento, el perfil antropométrico y el perfil social de estos atletas. Los resultados muestran que los velocistas y los saltadores fueron predominantemente mesomórficos. Los fondistas nacionales fueron meso-ectomórficos. Se observó que a medida que el nivel de rendimiento aumenta, los velocistas disminuyen sus cantidades de masa grasa. En los saltadores, con el aumento del nivel competitivo, la masa grasa y la masa de piel disminuyen. Los fondistas nacionales poseen una menor adiposidad subcutánea comparada con la de los velocistas. Comparado con otras pruebas (velocidad, saltos y fondo de nivel nacional), los lanzadores poseen características antropométricas más grandes y un mayor peso corporal. Los atletas juveniles peruanos pertenecen principalmente al nivel socioeconómico A. En conclusión, los atletas juveniles peruanos se encuentran divididos o categorizados morfológica y socioeconómicamente. Los atletas peruanos que se aproximen a las características morfológicas y sociales de los atletas internacionales y olímpicos estarán en mejores posibilidades de alcanzar el éxito a un alto nivel.

Descriptores: atletas, somatotipo, composición corporal, proporcionalidad, estatus socioeconómico.

\begin{abstract}
The aim of this study was to determine the anthropometric profile $(n=82)$ : body shape, body composition and proportionality, and the social profile $(n=71)$, socioeconomic status, of high level Peruvian junior athletes according to their level of performance (national and international level). It also establishes correspondences between athletics events, level of performance, the anthropometric profile and the social profile of these athletes. The results show that the sprinters and jumpers were predominantly mesomorphic. National long distance runners were mesoectomorphic. It was noted that while the performance level increases, the sprinters decrease their amount of body fat. In the jumpers, the fat mass and skin mass decrease while the competitive level increases. National long distance runners have a lower subcutaneous adiposity compared to the sprinters. Compared with other events (national sprinting, national jumping and national long distance running), the throwers are physically larger and heavier. Peruvian junior athletes mainly belong to the socioeconomic level A. In conclusion, the Peruvian junior athletes are divided or categorized morphologically and socioeconomically. Peruvian athletes that are close to the morphological and social characteristics of international and olympic athletes will be better chances to succeed at the high level.
\end{abstract}

Keywords: athletes, somatotype, body composition, proportionality, socioeconomic status.

\section{INTRODUCCIÓN}

La Cineantropometría y la Sociología nos han mostrado que hay factores morfológicos y sociológicos implicados en el rendimiento deportivo y que estos juegan a veces un papel principal en el éxito atlético. Lo que se sugiere es que el perfil antropométrico y el perfil social están involucrados en el éxito deportivo, las razones son las siguientes: primero, la carencia de un físico y de una complexión física adecuada a las exigencias del deporte pueden hacer prácticamente imposible lograr el éxito deportivo, sobre todo el de alto nivel [1]. Además, sabemos que hay características antropométricas propias para las carreras de media y larga distancia, y que estas están relacionadas considerablemente con el rendimiento de los corredores [2], [3]. Segundo, parece ser que las condiciones sociales y culturales condicionan la práctica de un deporte como el atletismo. Los resultados de Onywera et al. [4] indican que los corredores kenianos de élite se encuentran preponderantemente motivados por ganar dinero en las carreras. Lo más probable es que el bajo nivel 
socioeconómico (variable social) junto con las motivaciones de estos atletas (variable psicológica) los hacen elegir al atletismo como un medio para mejorar su situación de pobreza. Prueba de ello es que los países del este de África, por ejemplo, Kenia, en donde el promedio de salario diario es de $\$ 1-2$ [5], se han convertido en centros de producción de corredores de larga distancia [6]. Dicho en otros términos, las condiciones sociales y económicas parecen iniciar el proceso de participación deportiva, selección de una minoría y el éxito económico y deportivo de unos pocos.

Pese a que poco se conoce de las condiciones socioeconómicas de los atletas peruanos, una mirada al podio de los medallistas nacionales indica que los velocistas y saltadores pertenecen a niveles socioeconómicos más elevados que los fondistas y lanzadores. Esta situación nos lleva a describir el hecho de que los atletas peruanos poseen un perfil antropométrico y perfil social característico. En ese sentido, nos propusimos determinar detalladamente cada uno de estos perfiles según las especialidades atléticas y el nivel de rendimiento de los atletas peruanos juveniles de alto nivel, así como establecer correspondencias entre estas variables. Adicionalmente y con fines de control, se ha incluido una breve descripción de la procedencia geográfica de los atletas. El conocimiento de estos perfiles resaltaría su contribución sobre el éxito atlético, además de mostrar las diferencias entre la población general y los atletas de élite.

\section{MÉTODOS}

Acerca de la muestra y su división

La población comprende los atletas juveniles peruanos participantes en el XI Campeonato Nacional Juvenil de Atletismo 2007, realizado en la Villa Deportiva Nacional. La muestra fue no probabilística o dirigida [7], los criterios de inclusión fueron ser participante (varón o mujer) en las pruebas de dicho campeonato y haber ocupado uno de los cinco primeros puestos en cada prueba.

Los atletas evaluados para el perfil antropométrico y el perfil social fueron 82 y 71 atletas, respectivamente. Todas las evaluaciones fueron realizadas los días 1, 2, y 3 de Junio de 2007 durante el mencionado campeonato. Previamente, los atletas fueron informados acerca de los objetivos y procedimientos de las evaluaciones luego dieron su consentimiento voluntario para participar en el proyecto. Los evaluadores y el equipo de asistentes estuvieron informados y siguieron las normas $y$ pautas éticas descritas en el Código de Nürenberg y la Declaración de Helsinki.

Es necesario mencionar que por la naturaleza de este campeonato, que reúne a los mejores atletas peruanos juveniles en cada una de las pruebas, los mencionados deportistas tenían un entrenamiento previo que en algunos casos alcanzaba los tres años (información obtenida por comunicación personal); sin embargo, no nos fue posible determinar con exactitud el tiempo de entrenamiento de cada atleta. La muestra se dividió según sexo, tipos de eventos atléticos y niveles de rendimiento. Los eventos atléticos considerados para nuestro análisis fueron: velocidad $(100,200$ y 400 metros planos), medio fondo (800 y 1500 metros planos), fondo (3000, 5000 y 10000 metros planos), lanzamientos (bala, disco y martillo) y saltos (largo, tripe, alto y pértiga).

El $36.5 \%$ de atletas fueron provenientes del departamento y provincia de Lima y el $14.1 \%$ provino del departamento y provincia de Arequipa esto muestra una alta concentración de atletas entorno a la capital. Asimismo, el $11.8 \%$ de atletas vivía a una altura superior a los $3000 \mathrm{msnm}$ (Ancash, Cusco y Junín) y el $65.9 \%$ de ellos vivía a una altura menor a los 600 msnm (Ica, La Libertad, Lima, Tacna) (Tabla 2).

El rendimiento se determinó según las marcas obtenidas por los atletas en el XI Campeonato Nacional Juvenil del año 2007, de esta manera, la muestra se dividió en atletas de nivel nacional e internacional. Los atletas de nivel nacional son todos aquellos que participaron en el campeonato mencionado; y los atletas de nivel internacional son aquellos que además de participar en este campeonato, obtuvieron la marca mínima para competir en los Campeonatos Sudamericano Juvenil, Panamericano Juvenil y Mundial de Menores del año 2007.

Los perfiles antropométricos se determinaron siguiendo las normas de la International Society for the Advancement of Kinanthropometry [8]. El cálculo del somatotipo se realizó de acuerdo con el método de Heath y Carter [9]. La composición corporal fue determinada según el modelo de cinco componentes en el nivel tisular de Kerr [10]. Para la determinación de la proporcionalidad se utilizó la estratagema Phantom [11].

\section{Determinación del perfil social}

El perfil social así como su índice de estatus socioeconómico se determinó con un cuestionario socioeconómico adaptado de estudios previos [12]. 
Tabla 1. Resumen de la cantidad de atletas y su división.

\begin{tabular}{lcccc}
\hline Especialidades & \multicolumn{2}{c}{ Atletas nacionales } & \multicolumn{2}{c}{ Atletas internacionales } \\
& Varones & Mujeres & Varones & Mujeres \\
\hline Velocidad & 6 & 19 & 3 & 1 \\
Medio fondo & 4 & 7 & 1 & 1 \\
Fondo & 9 & 0 & 0 & 1 \\
Lanzamientos & 3 & 21 & 0 & 1 \\
Saltos & 4 & 2 & 2 & 1 \\
Total bruto & 26 & 49 & 6 & 5 \\
\hline
\end{tabular}

Tabla 2. Resumen de la procedencia geográfica de los atletas, dividida por departamentos (los signos $\widehat{O}$ $y$ o representan varones y mujeres, respectivamente).

\begin{tabular}{|c|c|c|c|c|c|c|c|c|c|c|c|}
\hline \multicolumn{2}{|c|}{ Departamentos } & 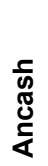 & $\begin{array}{l}\frac{\pi}{2} \\
\frac{3}{2} \\
\frac{d}{\alpha}\end{array}$ & 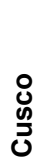 & 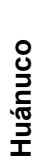 & $\underline{\mathbb{O}}$ & 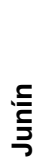 & 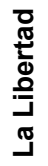 & 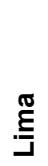 & $\stackrel{\circ}{\grave{J}}$ & 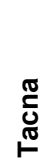 \\
\hline Velocidad & $\begin{array}{l}1 \\
0 \\
0\end{array}$ & & $\begin{array}{l}2 \\
4\end{array}$ & & 2 & 4 & & 2 & $\begin{array}{l}4 \\
9\end{array}$ & & 1 \\
\hline Medio fondo & $\begin{array}{l}+ \\
\hat{0} \\
0 \\
0\end{array}$ & 1 & $\begin{array}{l}3 \\
2\end{array}$ & 2 & & 1 & 2 & 1 & 1 & & \\
\hline Fondo & $\begin{array}{l}\hat{0} \\
0 \\
0\end{array}$ & 2 & 1 & 1 & 1 & 1 & 2 & & 1 & 1 & \\
\hline Lanzamientos & $\begin{array}{l}1 \\
0 \\
0\end{array}$ & & & & 1 & $\begin{array}{l}1 \\
2\end{array}$ & & 5 & $\begin{array}{c}2 \\
10\end{array}$ & & 4 \\
\hline Saltos & $\begin{array}{l}1 \\
0 \\
0 \\
0\end{array}$ & & & & 2 & 1 & & & $\begin{array}{l}3 \\
1 \\
\end{array}$ & & $\begin{array}{l}1 \\
1\end{array}$ \\
\hline Total bruto & & 3 & 12 & 3 & 6 & 10 & 4 & 8 & 31 & 1 & 7 \\
\hline
\end{tabular}

Determinación del perfil antropométrico

\section{RESULTADOS Y DISCUSIÓN}

\section{Perfil antropométrico}

\section{Velocistas}

La comparación entre nuestros velocistas varones nacionales $\mathrm{e}$ internacionales con respecto a los velocistas argentinos [13] y a los velocistas de las olimpiadas de Montreal 1976 [14] mostró que todos estos atletas fueron meso-ectomórficos. Asimismo, nuestros velocistas internacionales fueron menos endomórficos que el resto de este grupo de comparación, los velocistas nacionales por su parte obtuvieron el mayor ectomorfismo en este grupo. De acuerdo con este tipo de competición, se esperaba que los velocistas sean preponderantemente mesomórficos [15], es decir, que tengan una preponderancia hacia la robustez músculoesquelética relativa a la altura, hecho que también se corrobora en nuestros resultados.
Tabla 3. Somatotipos de los velocistas peruanos nacionales e internacionales. Los datos para las comparaciones fueron extraídos de [13], [14].

\begin{tabular}{llll}
\hline Nacionales & Internacionales & $\begin{array}{l}\text { Atletas } \\
\text { argentinos }\end{array}$ & $\begin{array}{l}\text { Atletas } \\
\text { olímpicos } \\
\text { de Montreal } \\
\mathbf{1 9 7 6}\end{array}$ \\
\hline $1.7-4.5-3.5$ & $1.3-4.1-3.4$ & $1.9-5.2-2.5$ & $1.7-5.2-2.8$ \\
Meso- & Meso- & Meso- & $\begin{array}{l}\text { Meso- } \\
\text { ectomorfos }\end{array}$ \\
\hline
\end{tabular}

A pesar del bajo endomorfismo de los velocistas internacionales peruanos (1.3), la comparación de su porcentaje graso $(\% \mathrm{FM}=20.1)$ con los velocistas de $100 \mathrm{~m}$ y $200 \mathrm{~m}$ que participaron en las olimpiadas de México 1968 (\%FM=12.4) [16] muestra que los velocistas peruanos tuvieron $7.7 \%$ más grasa corporal que los atletas olímpicos. En cuanto a la masa muscular, los velocistas nacionales peruanos $(\% \mathrm{MM}=48.1)$ difieren en $1.66 \%$ del porcentaje muscular de los velocistas estudiados por Berral et al. [17], quienes registraban un $49.76 \%$ para este componente. Este porcentaje muscular es similar al de los velocistas internacionales de este estudio (\%MM=49.4). Por tanto, podemos observar que la cantidad de músculo en el cuerpo aumenta con el nivel de rendimiento.

Los perfiles de proporcionalidad de los velocistas nacionales muestran diferencias considerables en los grosores de sus pliegues cutáneos, perímetros y longitudes óseas, lo que indica ciertas diferencias sustantivas en la distribución de la grasa del tronco y diferencias en la longitud de sus miembros inferiores, las mismas que tienen una contribución importante en el rendimiento en las carreras (Véase las figuras 3 y 4 ).

En el caso de los velocistas varones nacionales e internacionales, el tener de pliegues cutáneos muy cercanos o por debajo de -2 puntuaciones z puede ser considerado como un predictor importante de su rendimiento esto porque, en el sistema Phantom, una puntuación negativa, como -2, indica bajos valores para los pliegues cutáneos y esta característica ha mostrado ser beneficiosa para obtener un elevado rendimiento en cualquier distancia [18]. Otro punto que queda claro es que los velocistas poseen un mayor volumen corporal comparado con los fondistas y esto se puede apreciar en las diferencias en sus perímetros corporales así como en sus porcentajes de masa muscular y masa grasa. 


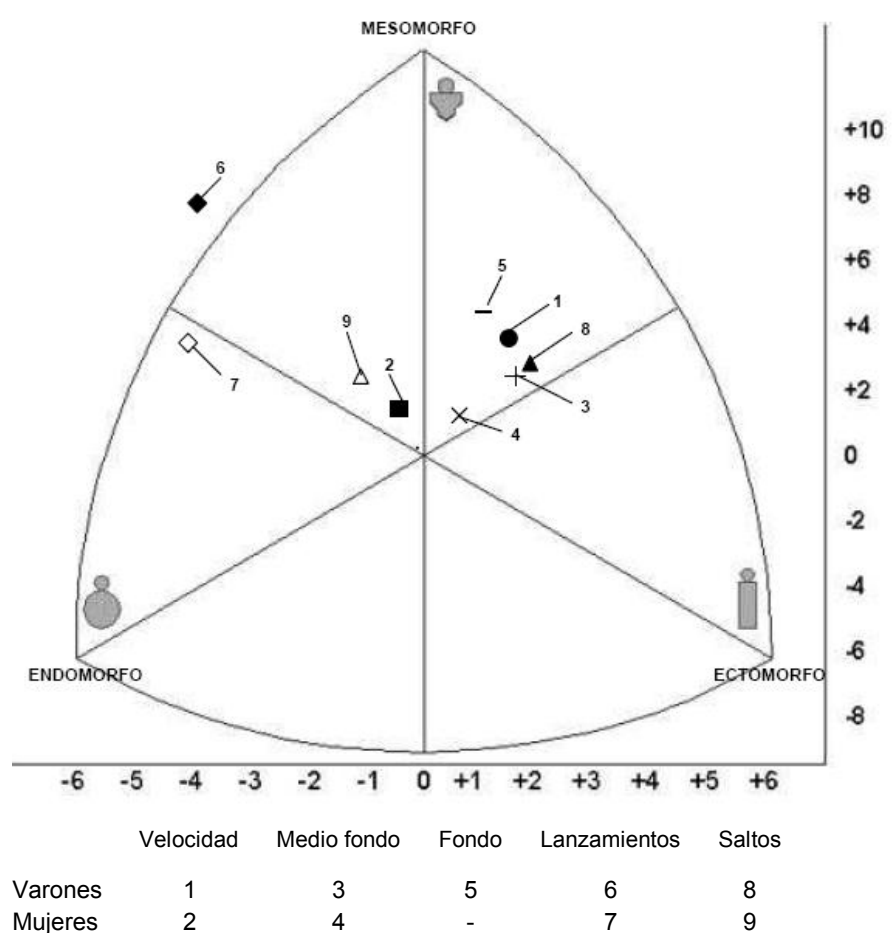

Figura 1. Los somatotipos de los atletas peruanos juveniles de nivel nacional (varones y mujeres).

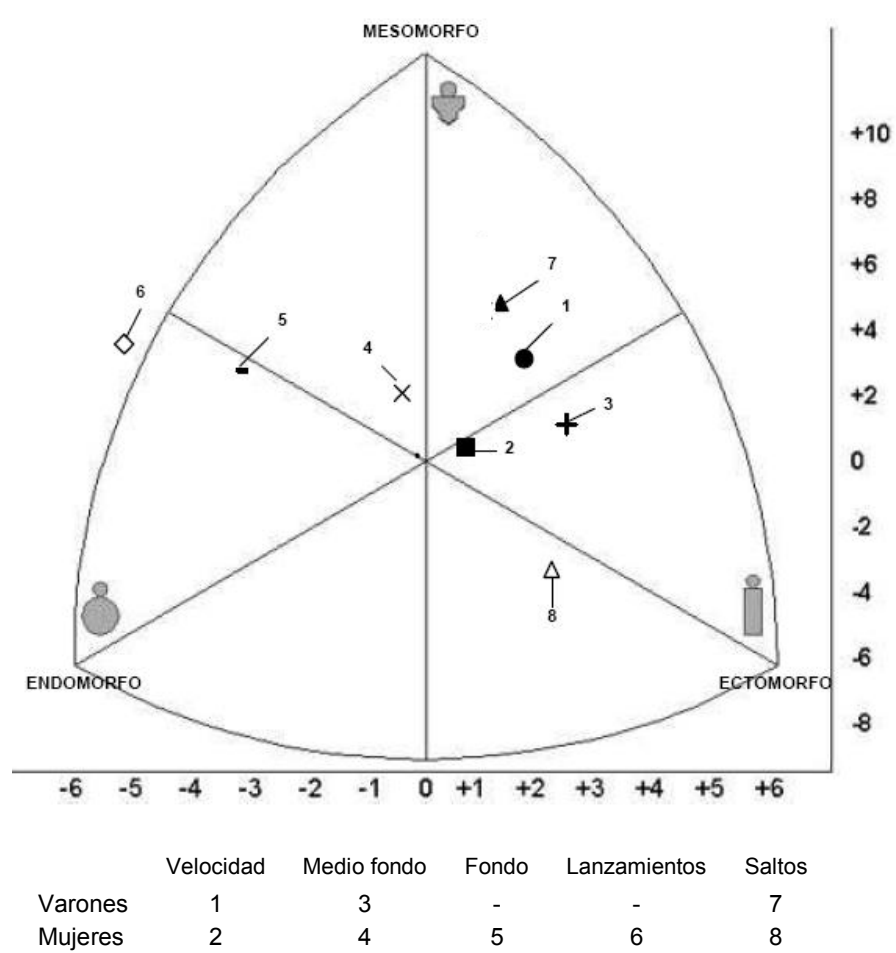

Figura 2. Los somatotipos de los atletas peruanos juveniles de nivel internacional (varones y mujeres).
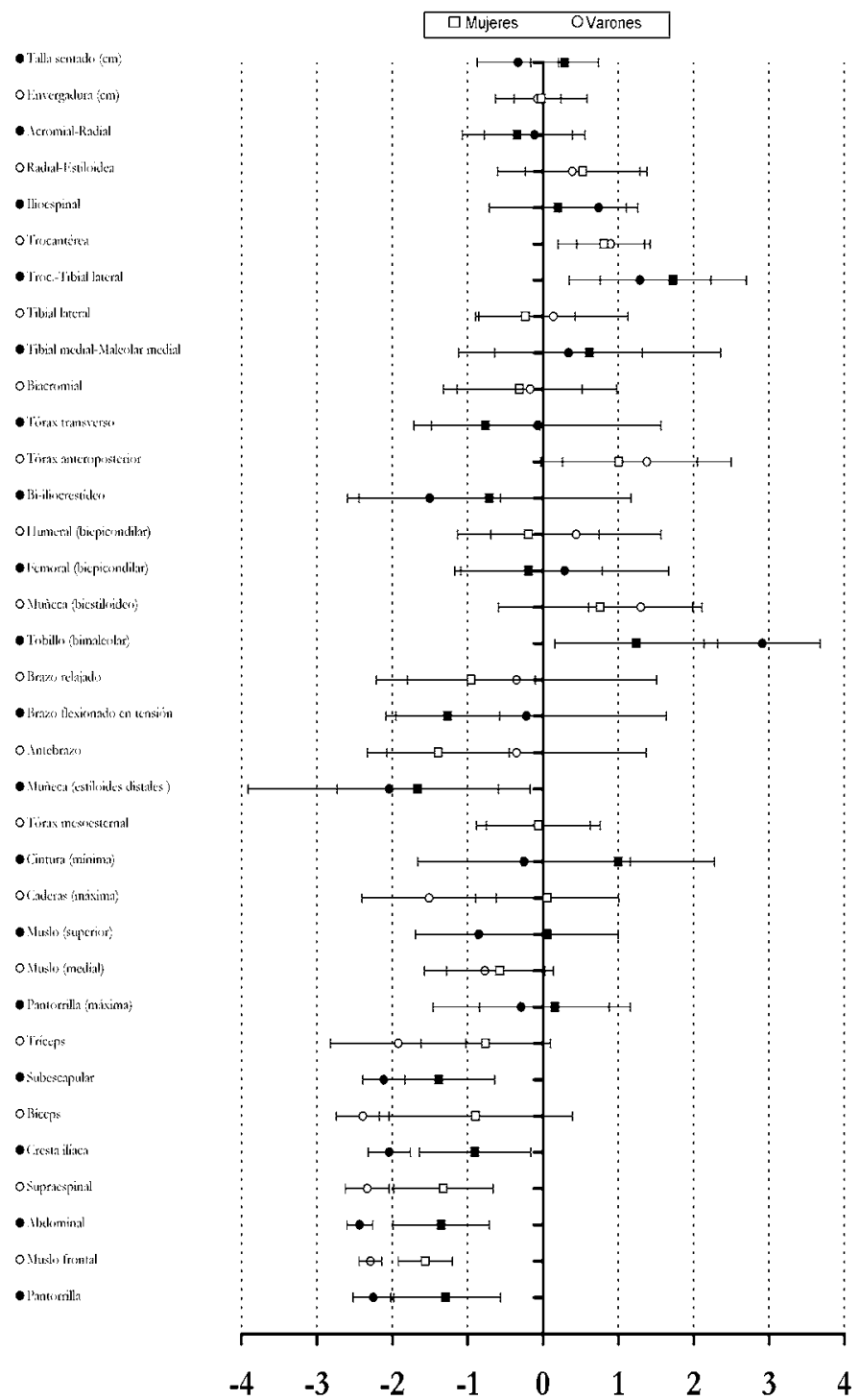

Figura 3. Perfil de proporcionalidad de los velocistas varones y mujeres de nivel nacional.

\section{Fondistas}

Los fondistas nacionales, somatotipo igual a 1.5-4.52.8, (de los cuales 2 de 10 vivían en provincias con una altitud menor que $521 \mathrm{msnm}$ mientras el resto residía a una altitud entre los 1894 hasta los 3827 


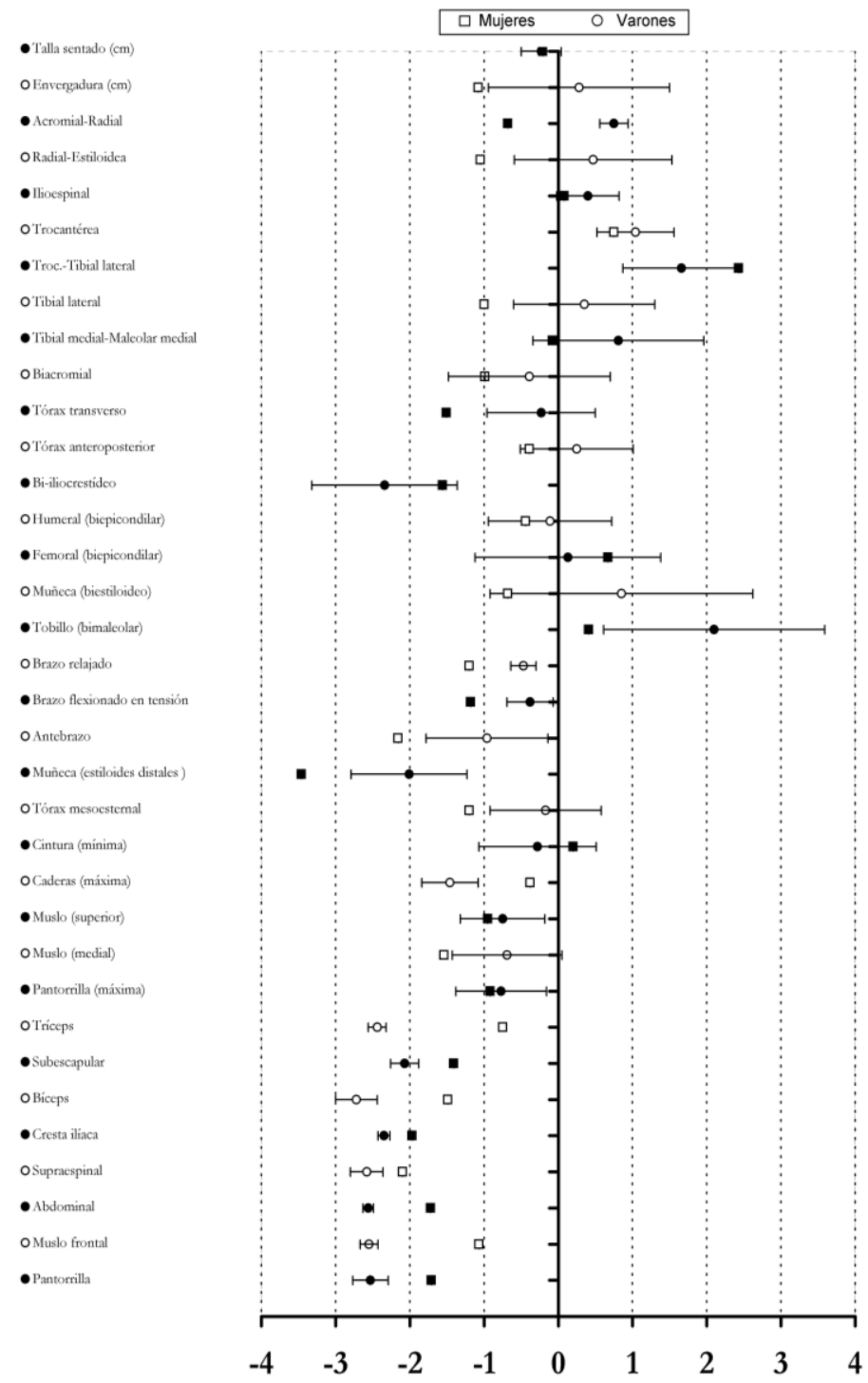

Figura 4. Perfil de proporcionalidad de los velocistas varones y mujeres de nivel internacional.

msnm) fueron más mesomórficos y menos ectomórficos que los corredores de larga distancia de los Juegos Africanos de 1995 y 2003 (1.6-2.7-4.2) [19]. La masa grasa de los fondistas nacionales peruanos $(\% \mathrm{FM}=20.1)$ fue mucho mayor que la obtenida por los corredores kenianos de larga distancia que tienen un entrenamiento de alta velocidad $(\% \mathrm{FM}=6.6)$ [20] y por los corredores sudafricanos de distancia $(\% \mathrm{FM}=8)$ [21]. La masa muscular de nuestros fondistas nacionales (\%MM=45) fue menor que la de los fondistas sudamericanos de élite $(\% \mathrm{MM}=48.72)$ [17]. Esta comparación muestra que si los corredores de larga distancia nacional tendrían menos masa adiposa y más masa muscular, entonces tendrían mejores posibilidades de alcanzar el éxito a nivel internacional.

Una comparación de los fondistas nacionales (Figura 5) con los velocistas nacionales e internacionales
(Figuras 3 y 4 ) muestra que hay una tendencia a disminuir el grosor de los pliegues cutáneos a medida que la distancia recorrida se hace más grande, hecho que se reitera cuando se compara la masa adiposa de los velocistas y fondistas nacionales $(\mathrm{FM}=13.6 \mathrm{~kg}, \quad \% \mathrm{FM}=21.6$ versus $F M=10.4$ kg, \%FM=20.1). Legaz y González [18] han mostrado que entre atletas españoles de élite, todas las medidas de los pliegues cutáneos fueron más bajas en los corredores de maratón comparados con los otros. Asimismo, esta tendencia está apoyada por la proporcionalidad de los corredores olímpicos de larga distancia, cuyos pliegues del tríceps, subescapular y suprailiaco fueron negativos con respecto de los valores de referencia del Phantom [11].
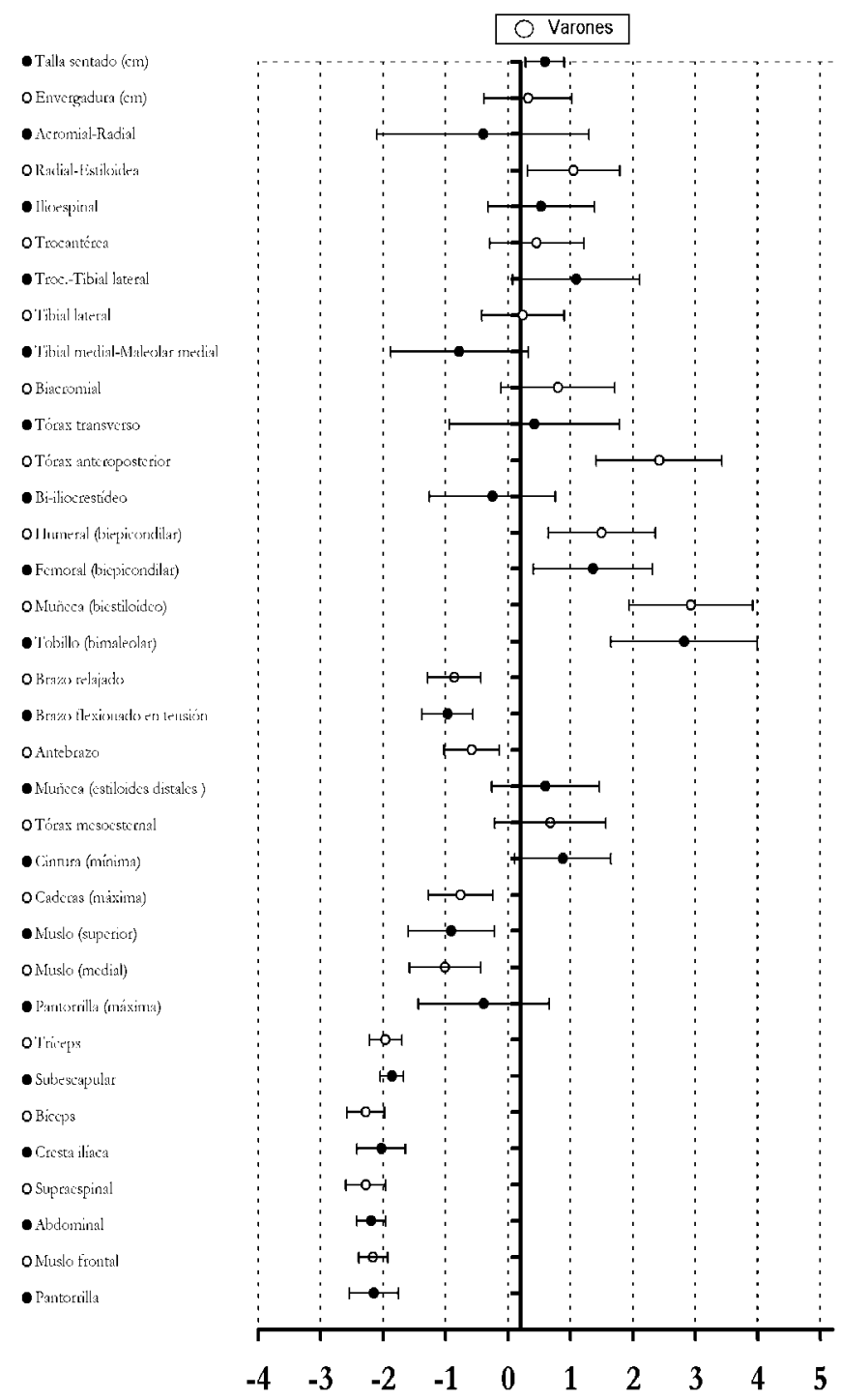

Figura 5. Perfil de proporcionalidad de los fondistas varones de nivel nacional. 


\section{Lanzamientos}

Los lanzadores nacionales (5.1-7.2-0.8) fueron más endomórficos que los lanzadores italianos de nivel nacional (2.9-5.9-1.1) [22], que los lanzadores de los XII Juegos Panamericanos (3.45-7.6-1.20) [23] y que los lanzadores argentinos (3.4-5.6-1.2) [13].

Un procedimiento sugerido para evaluar la importancia de los indicadores antropométricos muestra que cuando el coeficiente de variación de una variable antropométrica es menor en una población deportiva, más importante es esta variable en la contribución del éxito deportivo [24]. Así tenemos que la baja variabilidad del mesomorfismo (C.V.=14.4) indica que este componente posee una importancia considerable en esta prueba. Esto puede deberse a que en estos eventos existe un predominio de la fuerza así como de la técnica. Por lo tanto, los lanzadores nacionales tienen en el mesomorfismo una ventaja morfológica potencial. Desde este mismo punto vista, su elevado coeficiente de variabilidad para el ectomorfismo (C.V.=151.3) indica que este es un predictor del rendimiento que tiene escaza contribución en esta especialidad.

En general, el físico, como conjunto de rasgos antropométricos, de los lanzadores es más grande y pesado que en otras especialidades, considérese, por ejemplo, los velocistas nacionales (talla $=175.05$ $\mathrm{cm}$, peso $=64.43 \mathrm{~kg}, \mathrm{BMl}=21.03 \mathrm{~kg} / \mathrm{m}^{2}$ ), los fondistas nacionales (talla $=161.76 \mathrm{~cm}$, peso 54.16 , BMl $=20.7$ $\mathrm{kg} / \mathrm{m}^{2}$ ), saltadores nacionales (talla $=174.2$, peso $=62.12, \mathrm{BMI}=20.5 \mathrm{~kg} / \mathrm{m}^{2}$ ) versus los lanzadores nacionales (talla $=177.66 \mathrm{~cm}$, peso $=99.46 \mathrm{~kg}$, $\mathrm{BMI}=31.51 \mathrm{~kg} / \mathrm{m}^{2}$ ).

Tabla 4. Resumen de la composición corporal de los lanzadores peruanos nacionales. Los datos para las comparaciones fueron extraídos de [25].

\begin{tabular}{lll}
\hline Componentes & Nacionales & $\begin{array}{l}\text { Lanzadores } \\
\text { británicos } \\
\text { de élite }\end{array}$ \\
\hline \%FM & 27.6 & 16 \\
\%MM & 44.8 & - \\
\hline
\end{tabular}

La tenencia de envergaduras por encima de la puntuación 0 del sistema Phantom parece ser un factor común en nuestros lanzadores, lo que es compatible con los datos de los lanzadores olímpicos blancos [11]. Esta característica parece ser un factor beneficioso para el rendimiento en los lanzamientos; sin embargo, para investigaciones futuras haría falta probar esta presunta ventaja morfológica.
Saltos

Nuestros saltadores internacionales (1.2-4.8-2.8) fueron menos endomórficos y más mesomórficos que los saltadores de la olimpiada de Montreal 1976 (1.7-4.6-3.4) [14]. El porcentaje graso de nuestros saltadores internacionales $(19.1 \%)$ fue mayor que el registrado en los saltadores olímpicos de Tokio 1964 $(14.1 \%)$ y que el observado en los saltadores de triple salto de México 1968 (8.4\%) [16].

Algunas características antropométricas adicionales, como el BMl y la masa de piel, muestran que los saltadores internacionales fueron más pesados que los nacionales. Asimismo, ellos tuvieron una menor masa de piel (SM) con respecto a los nacionales ( $\% S M=5.9$ versus $\% S M=6.3)$. Considerando las ecuaciones de Kerr [10], podemos afirmar que una menor masa de piel es un indicador de una menor superficie corporal. Este dato es relevante para la biomecánica del salto, puesto que a menor superficie corporal es menor la resistencia ejercida por el aire en la fase de vuelo.

En suma, en las pruebas de saltos, algunos factores morfológicos como el bajo peso relativo, una tendencia hacia el mesomorfismo, una menor superficie corporal, un elevado porcentaje muscular y un bajo porcentaje graso parecen ser características propias en estos atletas.

\section{Perfil social}

\section{Estatus socioeconómico}

De acuerdo con los datos de este estudio, podemos decir que el atletismo peruano juvenil de élite es una actividad social dirigida principalmente al nivel socioeconómico $A$, el mismo que en una cantidad considerable está compuesto por velocistas (Figuras 6.A y 6.B). La participación en el atletismo juvenil competitivo en muchos casos conlleva una preparación que requiere varios años, así como ciertas condiciones, equipamientos e indumentarias que demandan de la ayuda directa y el apoyo de los padres, situación que es muy similar a la que ocurre con los jóvenes de la población general cuando se trata de su participación en alguna modalidad de actividad física [26]. Nuestros datos muestran que 6 de 71 atletas trabajaban temporalmente a la fecha de la recolección de datos para este estudio, pero que ninguno de ellos era velocista. Esto muestra que los corredores de distancias cortas, al pertenecer a niveles socioeconómicos más altos, tienen un poder adquisitivo mayor y no necesitan trabajar dado que sus necesidades básicas y algunas secundarias, como la de participar activamente en el sistema deportivo, están cubiertas. 
En ese sentido, el significado del deporte para los atletas de más elevado estatus socioeconómico parece ser diferente con respecto al de los atletas de otros estratos sociales. Pensamos que la práctica de este deporte y en especial la participación en pruebas de corta duración son un signo o indicador de clase social para estos deportistas y sus padres. Esta idea es plausible con lo mostrado por Lever [27], en donde los miembros de clubes de fútbol de Rio de Janerio y Sao Paulo pertenecían a niveles sociales más elevados que los clubes de provincias. Según Lever [27], la afiliación a un club de la clase alta no es necesariamente ocasionada por el deseo de ganar dinero tal como ocurre en los clubes de condición más modesta, sino más bien por el deseo de satisfacer la necesidad de recrearse.

Nuestros datos también muestran que por lo menos el atletismo nacional juvenil de élite, considerado como un deporte, no es una actividad exclusiva de la clase ociosa o del estrato más elevado como afirmaba Thorstein Veblen [28], sino que cuenta con una amplia difusión en los estratos A, B y C.

Por otro lado, parece ser que una mejoría del estatus socioeconómico va acompañada, aunque no
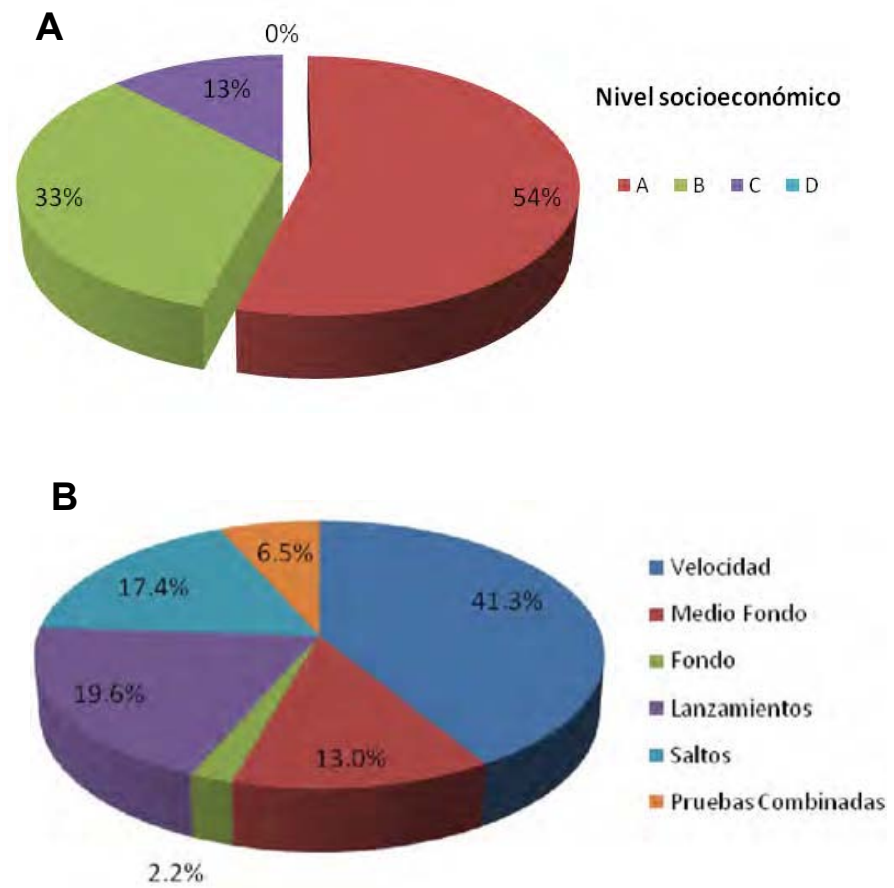

Figura 6: A. El estatus socioeconómico expresado como porcentajes del número de atletas peruanos juveniles según sus especialidades (ambos sexos). 6.B. Composición porcentual del nivel socioeconómico $A$ según especialidades (ambos sexos).

necesariamente causada, por un aumento del nivel competitivo de algunos atletas (Figura 7.A y 7.B). Hemos observado que los velocistas, mediofondistas y saltadores internacionales tienden a poseer un mejor nivel socioeconómico que sus pares de nivel nacional, tendencia que es compartida con las mediofondistas. Por ello, es probable que un indicador social del alto rendimiento atlético, actual o potencial, en este tipo de eventos sea la pertenencia a un nivel socioeconómico elevado; en particular, la pertenencia a un nivel social medio-medio o medioalto. Sin embargo, el caso de las lanzadoras es algo diferente porque ellas tienden a permanecer en el nivel socioeconómico $B$ a medida que aumenta el nivel competitivo.

Hasta aquí, sabemos que hay una estratificación social en el atletismo y que esta está vinculada con el tipo de evento. Comparativamente, quienes logran el éxito atlético son aquellos que están en mejores condiciones morfológicas y sociales. Adicionalmente, se sabe que el número de los deportistas que alcanza el éxito es muy reducido, comparado con la gran masa que está involucrada directamente en el deporte [29]. Sin embargo, queda una lista de problemas que faltan resolver como qué es lo que origina y cómo se produce la movilidad social en el atletismo, qué aspiran lograr con el atletismo y qué es lo que realmente logran estos deportistas. Por estas razones, en futuras investigaciones será necesario enfocar la atención en la explicación de la movilidad social en el atletismo y, de manera más general, en el deporte. Para ello será necesario estudiar, entre otros, las aspiraciones, las preferencias y las motivaciones, individuales y

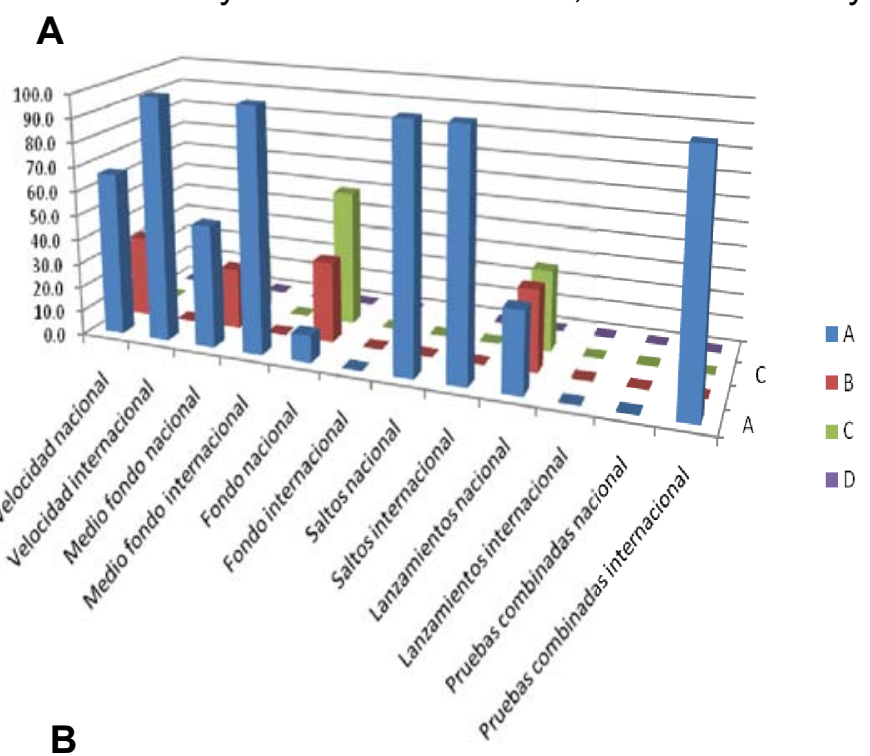




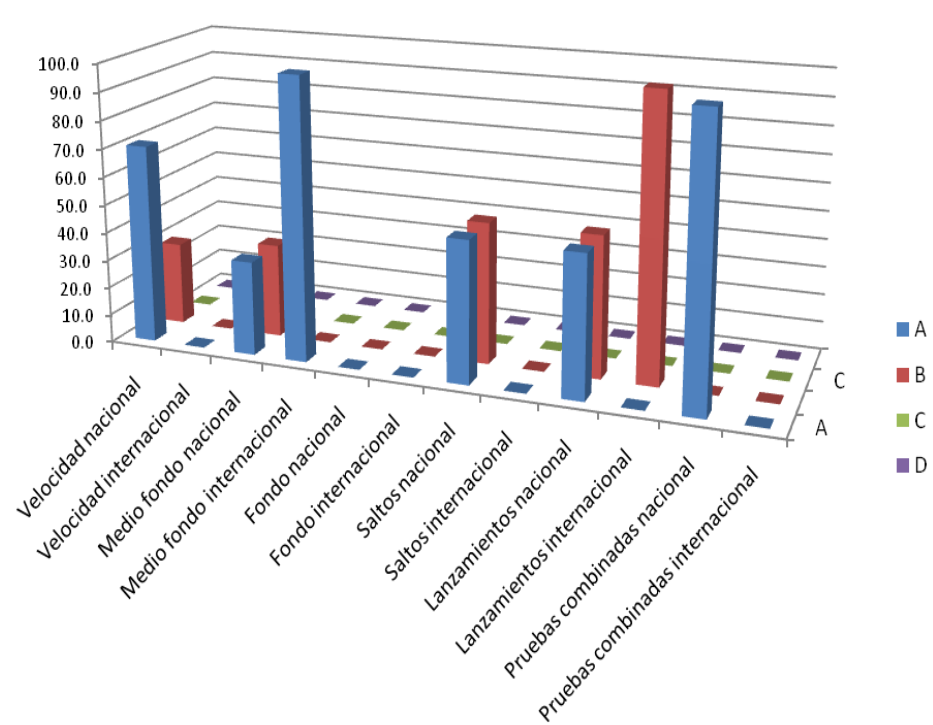

Figura 7. El estatus socioeconómico de los atletas peruanos juveniles (7.A. Varones, 7.B. Mujeres) en porcentajes, por especialidades y nivel de rendimiento.

sociales, que son las que guían, por lo menos en parte, la acción social. Esto último es deseable porque cada una de estas debería estar fundamentada tanto ética como científicamente y por esa razón hay que investigarlas. Las consecuencias para la tecnología social y la política son evidentes porque todo agente social elabora planes, plantea políticas y se propone regular o transformar algún aspecto de la realidad social, y es justamente esto lo que no se puede hacer eficazmente cuando se abraza el mito y el prejuicio en vez de la hipótesis científica y el dato bien fundado.

\section{CONCLUSIONES}

Finalmente, este trabajo muestra que los atletas juveniles peruanos se encuentran divididos 0 categorizados morfológica y socioeconómicamente de acuerdo con su especialidad atlética y nivel de rendimiento. Asimismo, antropométrica y socialmente, los atletas peruanos que se aproximen a las características de los atletas internacionales y olímpicos estarán en mejores posibilidades de alcanzar el éxito a un alto nivel.

\section{AGRADECIMIENTOS}

Deseo agradecer al Lic. Polo Mayorca (UNMSM, Perú) con quien he discutido mis puntos de vista acerca de los fundamentos de la composición corporal y quien me ha alentado a seguir estudiándolos. Asimismo, agradezco las atenciones y el tiempo que me brindaron el Dr. Galo Narváez (Universidad de Catamarca, Argentina), Dr. Carlos
Benitez (Universidad de La Plata, Argentina) y Francis Holway (Cineantropometría ISAK Nivel 4 y Consejo Ejecutivo ISAK, Argentina) para la revisión de los borradores de la tesis del autor de este artículo, la misma que he tratado de exponer de forma resumida en este documento. Debo enfatizar que los errores que el lector pueda encontrar son de mi estricta responsabilidad.

\section{REFERENCIAS}

[1] L. Carter, "Morphological factors limiting human performance", en: D. H. Clarke, H. M. Eckert. Eds., Limits of Human Performance, American Academy of Physical Education Papers, Vol. 18 (Human Kinetics, Illinois, 1985).

[2] P. Bale, D. Bradbury, E. Colley, Brit. J. Sports Med. 20(1986) 170-173.

[3] A. L. Arrese, E. S. Ostáriz, J. Sport Sci. 24(2006) 69-76.

[4] V. Onywera, R. Scott, M. Boit, Y. Pitsiladis, J. Sport Sci. 24(2006) 415-422.

[5] B. Fudge, Tesis de Doctorado: "Diet, Hydratation, Lifestyle and Training Practices of Elite Kenyan Endurance Runners", Glasgow University, 2009.

[6] J. Bale, J. Sang, "Out of Africa: The „Development' of Kenyan Athletics, Talent Migration and the Global Sports System", en: J. Bale, J. Maguirre. Eds., The Global Sports Arena (Frank Cass Publishers, London, 2003).

[7] R. Sampieri, C. Fernández, P. Baptista, "Metodología de la investigación" (McGraw Hill, México, 2006).

[8] K. Norton, N. Whittingham, L. Carter, D. Kerr, C. Gore, M. Marfell-Jones, "Técnicas de medición antropométrica", en: K. Norton, T. Olds. Eds., Antropométrica (Biosystem Servicio Educativo, Rosario, 2000).

[9] L. Carter, "Somatotipo", en: K. Norton, T. Olds. Eds., Antropométrica (Biosystem Servicio Educativo, Rosario, 2000).

[10] D. Kerr, Tesis de Maestría: "An Anthropometric Method for Fractionation of Skin, Adipose, Bone, Muscle and Residual Tissue Masses, in Males and Females Age 6 to 77 years", Simon Fraser University, 1988.

[11] W. D. Ross, R. Ward, "Proportionality of Olympic Athletes", en: L. Carter. Ed., Physical Structure of Olympic Athletes, Part II (Karger, Basel, 1984).

[12] J. Flores, "Perfil Socioeconómico, Cultural y Político del estudiante san marquino" (UNMSM, Lima, 1993).

[13] N. A. Lentini, J. M. Gris, M. L. Cardey, G. 
Aquilino, P. A. Dolce, PubliCE Standard. Pid: 738 (2006).

[14] L. Carter, "Somatotypes of Olympic Athletes from 1948-1976", en: L. Carter. Ed., Physical Structure of Olympic Athletes, Part II (Karger, Basel, 1984).

[15] P. F. Radford, "Sprinting", en: T. Reilly, N. Secher, P. Snell, C. Williams. Eds., Physiology of Sports (Taylor and Francis, London, 2005).

[16] A. L. De Garay, L. Levine, L. Carter, "Genetic and Anthropological Studies of the Olympic Athletes" (Academic Press, New York, 1974).

[17] F. J. Berral, A. Escribano, C. J. Berral, J. L. Lancho, E. H. De Rose, Actas del C.C.O. (1992) 230-237.

[18] A. Legaz, J. González, E. Serrano, J. Sports Med. Phys. Fitness. 45(2005) 512-517.

[19] C. Underhay, J. De Ridder, L. Amusa, A. Toriola, A. Agbonjinmi, J. Adeogun, A. J. P. H. E. R. D. 11(2005) 6-16.

[20] V. Billat, P. Lepretre, A. Heugas, M. Laurence, D. Salim, J. P. Koralsztein, Med. Sci. Sports Exerc. 35(2003) 297-304.

[21] S. Semple, N. Neveling, R. Roussouw, A. J. P. H. E. R. D. 9(2003) 238-249.

[22] F. Viviani, G. Casagrande, "Il somatotipo in atleti italiani (dati preliminari)", en: UAI, FIAI. Eds., Sommari dei Contributi Scientifici. IX Congreso degli Antropologi Italiani. (Adriatica Editrice, Bari, 1991).

[23] P. A. Cordero, S. Bossio, G. E. Narváez, "Somatotipos Panamericanos: Proyecto Antropométrico Panamericano PRAPANA 95" (Trabajo no publicado).

[24] K. Norton, T. Olds, S. Olive, N. Craig, "Antropometría y Performance Deportiva", en: K. Norton, T. Olds. Eds., Antropométrica (Biosystem Servicio Educativo, Rosario, 2000).

[25] D. Kidd, E. Winter, Brit. J. Sports Med. 17(1983) 152-153.

[26] J. Sallis, J. Prochaska, W. Taylor, Med. Sci. Sports Exerc. 32(2000) 963-975.

[27] J. Lever, "El fútbol en Brasil", en: G. Lüschen, K. Weis. Eds., La Sociología del Deporte (Miñón, Valladolid, 1976).

[28] T. Veblen, "Teoría de la clase ociosa" (Fondo de Cultura Económica, México, 2004).

[29] D. S. Eitzen, "Fair and Foul: Beyond the Myths and Paradoxes of Sport" (Rowman \& Littlefield, Maryland, 1999).

E-mail: americo.bravo@correo.unmsm.edu.pe 